\title{
Through-space and through-bridge components of chemical bonds
}

\author{
Roman F. Nalewajski
}

Received: 22 July 2010 / Accepted: 27 September 2010 / Published online: 21 October 2010 C The Author(s) 2010. This article is published with open access at Springerlink.com

\begin{abstract}
The direct (through-space) and indirect (through-bridge) components of chemical interactions between atomic orbitals are identified in both the Wiberg bondorder formalism and the Orbital Communication Theory of the chemical bond. The illustrative examples using the Hückel description of the conjugated $\pi$-bonds in benzene and butadiene are given and the existence of the through-bridge bond between bridgehead carbons in small propellanes is conjectured.
\end{abstract}

Keywords Bond orders · Chemical interactions · Direct/indirect bonding mechanisms · Entropic bond indices - Information-theoretic bond multiplicities . Orbital Communication Theory of chemical bond · Through-bridge/through-space bond components . Wiberg bond-orders

\section{Introduction}

In Molecular Orbital (MO) theory the chemical interaction between, say, two (valence) Atomic-Orbitals (AO) or general basis functions originating from different atoms is strongly influenced by their direct overlap/interaction, which conditions the bonding effect experienced by electrons occupying their bonding combination in the molecule, compared to the non-bonding reference of electrons on separated AO. This throughspace bonding mechanism is then associated with typical accumulation of the valence electrons in the region between the two nuclei, due to the constructive interference

Throughout the paper $A$ denotes a scalar quantity, $\boldsymbol{A}$ stands for a row-vector, and $\mathbf{A}$ represents a square or rectangular matrix.

R. F. Nalewajski (凶)

Theoretical Chemistry Department, Jagiellonian University, R. Ingardena 3, Cracow 30-060, Poland e-mail: nalewajs@chemia.uj.edu.pl 
between the two AO, which exhibits some polarization reflecting the initial electronegativity difference of the two atoms involved. In other words, such "shared" bond charge is synonymous with the presence of the bond-covalency in direct ("throughspace") interaction between the two AO, which is also reflected by the associated covalent Valence-Bond (VB) structure. Similar effect of the bonding accumulation of the information densities relative to the promolecular distribution is detected in maps of alternative densities of the entropy deficiency and displacement in Shannon's entropy [1-5]. Accordingly, the complementary bond-ionicity aspect is manifested in MO theory by the MO polarization, or alternatively - by the participation of the orthogonal part of the ionic VB structure in the ground-state wave function. Let us recall, that on the elementary CID-level of the Configuration-Interaction (CI) in the minimum basis set both MO and VB descriptions of the chemical bond in $\mathrm{H}_{2}$ are exactly equivalent, differing only in specific routes of arriving at the same two-electron ground-state wave function describing the singlet-paired electrons.

The inter-orbital bonding interaction lacking such an accumulation of the bond charge (information), e.g., in the smallest propellanes, can be also realized indirectly, through the neighboring AO intermediaries forming a "bridge" for an effective interaction between distant terminal AO, e.g., those located on bridgehead carbons in propellanes, or the meta- and para-carbons in the benzene ring [1,2,6,7]. This indirect ("through-bridges") mechanism reflects the implicit dependence between AO resulting from the overall participation of the AO-intermediaries in the system chemical bonds determined by the subspace of the occupied MO. The associated through-bridge bondorder of the central bond in [1.1.1] propellane has been estimated using the generalized quadratic Wiberg-type indices [8-11] from the two-electron difference approach [12-17] to be of the order of 0.8 bond, with the full, single-bond in [2.2.2] propellane including the additional 0.2 bond originating from the direct, through-space component $[1,2,6]$, which is clearly detected in both the density-difference, and local information-theoretic (IT) probes, e.g., the entropy-deficiency, entropy-difference diagrams [1-3], Electron Localization Function (ELF) [18-21], and Contra-Gradience (CG) plots [22-25].

Thus, such a generalized outlook on the bond-order concept $[1,2]$, emerging from both the Wiberg [8] or quadratic-difference approaches in MO theory [1,12-17] and the IT bond-multiplicity in the Orbital Communication Theory (OCT) of the chemical bond [2,7,26-29], identifies the chemical bond multiplicity as a measure of the statistical "dependence" (non-additivity) between orbitals on different atomic centers. On one hand, this dependence between basis functions of different atoms can be partly realized directly (through space), by the constructive interference of orbitals (probability amplitudes) on two atoms, which increases the electron density between them. On the other hand, it can also have an indirect origin, through the dependence on orbitals of the remaining Atoms-in-Molecules (AIM) used to construct the system occupied MO. The latter component is due to the orthonormality relations of the occupied MO, which determine the framework of chemical bonds in the molecule. Therefore, each pair of AO or AIM exhibits the partial through-space and through-bridge components: the bond-order of the former quickly vanishes with an increase of the inter-atomic separation or when the interacting $\mathrm{AO}$ are heavily engaged in forming other chemical bonds, while the latter can still assume appreciable values, when the remaining atoms 
form an effective bridge of the neighboring, chemically bonded atoms, which links the specified AO/AIM in question.

In the present analysis, which was prompted by the discussion at the international meeting "Twenty Years ELF" (Paris, June 21-24, 2010), we shall identify both these components of chemical interactions using the Wiberg [8] measure of bond multiplicities. The corresponding IT-covalencies will also be examined within the recently proposed OCT of the chemical bond [2,7,26-29].

\section{Bond projections and density matrix}

In standard SCF MO theory the network of chemical bonds is determined by the occupied MO in the system ground-state. Let us assume the closed-shell ( $c s$ ) configuration of $N=2 n$ electrons in the standard spin-restricted Hartree-Fock (RHF) description, which involves $n$ lowest, doubly-occupied (orthonormal) MO. In the familiar LCAO MO approach they are generated as linear combinations of the (Löwdin-orthogonalized) AO (basis functions) $\chi=\left(\chi_{1}, \chi_{2}, \ldots, \chi_{m}\right)=\left\{\chi_{i}\right\}$ contributed by the system constituent atoms, $\langle\chi \mid \chi\rangle=\left\{\delta_{i, j}\right\} \equiv \mathbf{I}$,

$$
\boldsymbol{\varphi}=\left[\left(\varphi_{1}, \varphi_{2}, \ldots, \varphi_{n}\right),\left(\varphi_{n+1}, \ldots \varphi_{m}\right)\right] \equiv\left(\varphi^{o}, \varphi^{v}\right)=\left\{\varphi_{s}\right\}=\chi \mathbf{C}=\chi\left(\mathbf{C}^{o} \mid \mathbf{C}^{v}\right),
$$

where the rectangular matrices $\mathbf{C}^{o}=\left\langle\chi \mid \varphi^{o}\right\rangle$ and $\mathbf{C}^{v}=\left\langle\chi \mid \varphi^{v}\right\rangle$ group the relevant expansion coefficients of the $n$ (doubly-occupied) and $m-n$ virtual (empty) MO, respectively, to be determined using the iterative self-consistent-field (SCF) procedure. The full LCAO MO matrix $\mathbf{C}$ is unitary, $\mathbf{C}^{\dagger}=\mathbf{C}^{-1}$, since it "rotates" orthonormal AO into the orthonormal MO, and hence the inverse transformation reads: $\chi=\varphi \mathbf{C}^{\dagger}$.

The molecular electron density,

$$
\rho(\boldsymbol{r})=2 \boldsymbol{\varphi}^{o}(\boldsymbol{r}) \boldsymbol{\varphi}^{o \dagger}(\boldsymbol{r})=\chi(\boldsymbol{r})\left[2 \mathbf{C}^{o} \mathbf{C}^{\sigma^{\dagger}}\right] \chi^{\dagger}(\boldsymbol{r}) \equiv \chi(\boldsymbol{r}) \gamma \chi^{\dagger}(\boldsymbol{r})=N p(\boldsymbol{r}),
$$

and hence also the one-electron probability distribution $p(\boldsymbol{r})=\rho(\boldsymbol{r}) / N$, the shapefactor of $\rho$, are determined by the 1-density matrix $\gamma$, also called the Charge-andBond-Order (CBO) matrix,

$$
\begin{aligned}
\boldsymbol{\gamma} & =2\left\langle\chi \mid \varphi^{o}\right\rangle\left\langle\varphi^{o} \mid \chi\right\rangle=2 \mathbf{C}^{o} \mathbf{C}^{o \dagger}=2\left\langle\chi\left|\hat{\mathrm{P}}_{\varphi}^{o}\right| \chi\right\rangle \\
& =2\left(\langle\chi| \hat{\mathrm{P}}_{\varphi}^{o}\right)\left(\hat{\mathrm{P}}_{\varphi}^{o}|\chi\rangle\right) \equiv 2\left\langle\chi^{b} \mid \chi^{b}\right\rangle=\mathbf{C d C}^{\dagger} \\
& =\left\{\gamma_{i, j}=2\left\langle i\left|\hat{\mathrm{P}}_{\varphi}^{o}\right| j\right\rangle=2\left\langle i\left|\left(\hat{\mathrm{P}}_{\varphi}^{o}\right)^{2}\right| j\right\rangle \equiv 2\left\langle i^{b} \mid j^{b}\right\rangle\right\},
\end{aligned}
$$

where the diagonal matrix $\mathbf{d}$ groups the MO occupations, $\mathbf{d}=\left\{\delta_{s, s^{\prime}}(2, s \leq n ; 0, s>\right.$ $n)\}$, and the basis set projections onto the occupied (bond) subspace $\varphi^{o}$,

$$
\left|\chi^{b}\right\rangle=\hat{\mathrm{P}}_{\varphi}^{o}|\chi\rangle=\left|\varphi^{o}\right\rangle\left\langle\varphi^{o} \mid \chi\right\rangle=\left|\varphi^{o}\right\rangle \mathbf{C}^{o \dagger}=\left\{\hat{\mathrm{P}}_{\varphi}^{o}|i\rangle=\left|i^{b}\right\rangle\right\},
$$


determine the so called bond-projections of AO.

It also follows from Eq. 3 that MO determine eigenvectors of $\gamma$ corresponding to eigenvalues (occupations) $\mathbf{d}: \boldsymbol{\gamma} \mathbf{C}=\mathbf{C d}$. Thus, $m$ bond-projections $\left|\boldsymbol{\chi}^{b}\right\rangle$ of AO effectively span the $n$-dimensional subspace of the occupied $\mathrm{MO},\left|\varphi^{o}\right\rangle=\left\{\left|s^{o}\right\rangle\right\}$. Indeed, the occupied MO determine the complete orthonormal basis in the bonding vector space $\left|\chi^{b}\right\rangle$, so that any bond-projection of AO can be expanded in $\left|\varphi^{o}\right\rangle$ [see Eq. 4].

The $\mathrm{CBO}$ matrix thus constitutes the $\mathrm{AO}$ representation of the projection operator onto the subspace of all doubly-occupied MO, $\gamma=2\left\langle\chi\left|\hat{\mathrm{P}}_{\varphi}^{o}\right| \chi\right\rangle$,

$$
\hat{\mathrm{P}}_{\varphi}^{o}=\left|\varphi^{o}\right\rangle\left\langle\varphi^{o}\left|=\sum_{s}^{o c c d}\right| \varphi_{s}\right\rangle\left\langle\varphi_{s}\left|\equiv \sum_{s}^{o c c d} \hat{\mathrm{P}}_{s}=1 / 2\right| \chi\right\rangle \gamma\langle\chi|, \quad\left(\hat{\mathrm{P}}_{\varphi}^{o}\right)^{2}=\hat{\mathrm{P}}_{\varphi}^{o}
$$

It thus satisfies the following idempotency relation

$$
(\gamma)^{2}=4\left\langle\chi\left|\hat{\mathrm{P}}_{\varphi}^{o}\right| \chi\right\rangle\left\langle\chi\left|\hat{\mathrm{P}}_{\varphi}^{o}\right| \chi\right\rangle=4\left\langle\chi\left|\left(\hat{\mathrm{P}}_{\varphi}^{o}\right)^{2}\right| \chi\right\rangle=4\left\langle\chi\left|\hat{\mathrm{P}}_{\varphi}^{o}\right| \chi\right\rangle=2 \gamma
$$

since in the adopted basis set $|\chi\rangle\langle\chi| \hat{\mathrm{P}}_{\varphi}^{o}=\hat{\mathrm{P}}_{\varphi}^{o}$ or $|\chi\rangle\langle\chi|=1$.

The 1-matrix reflects the promoted, valence state of AO in the molecule, with the diagonal elements measuring the effective electron occupations of basis functions, $\left\{N_{i}=\gamma_{i, i}=N p_{i}\right\}$, with probabilities $\boldsymbol{p}=\left\{p_{i}=\gamma_{i, i} / N\right\}$ of the basis functions occupancy in molecule: $(1 / N) \operatorname{Tr} \boldsymbol{\gamma}=\sum_{i} p_{i}=1$. The off-diagonal CBO elements between $\mathrm{AO}$ on different atoms similarly reflect the bonding status of the resultant interaction of the specified AO pair in the molecule, with the positive (negative) values signifying the bonding (anti-bonding) coupling between basis functions, and the vanishing bond-order $\gamma_{i, j}=0$ identifying the non-bonding net chemical interaction, i.e., $\left|i^{b}\right\rangle=0$ or $\left|j^{b}\right\rangle=0$. Thus, the "constructive" (bonding) interference between two AO, the basis functions of SCF MO calculations, requires the two AO to exhibit the positive product of their direct bond-projections, while the negative product value identifies their "destructive" interference in the molecular bond system.

Therefore, the (non-othonormal) bond-projected AO basis contains $m-n$ linearly dependent vectors, with 1-matrix determining the associated overlap matrix:

$$
\mathbf{S}=\left\langle\chi^{b} \mid \chi^{b}\right\rangle=\boldsymbol{\gamma} / 2=\mathbf{C}^{o} \mathbf{C}^{o \dagger}=\left\{S_{i, j}=\left\langle i^{b} \mid j^{b}\right\rangle\right\}
$$

The $\left|i_{b}\right\rangle$ projector can be expressed in terms of the re-normalized bond-projection of AO, onto $\left|\bar{\chi}_{i}^{b}\right\rangle=\left|i^{b}\right\rangle \sqrt{2 / \gamma_{i, i}},\left\langle\bar{\chi}_{i}^{b} \mid \bar{\chi}_{i}^{b}\right\rangle=1$,

$$
\hat{\overline{\mathrm{P}}}_{i}^{b}=\left|\bar{\chi}_{i}^{b}\right\rangle\left\langle\bar{\chi}_{i}^{b}|=| i^{b}\right\rangle 2 \gamma_{i, i}^{-1}\left\langle i^{b}\right|=2 \gamma_{i, i}^{-1} \hat{\mathrm{P}}_{i}^{b},\left(\hat{\overline{\mathrm{P}}}_{i}^{b}\right)^{2}=\hat{\overline{\mathrm{P}}}_{i}^{b}
$$


One also observes that the idempotent projection onto the whole bonding subspace $\left|\chi^{b}\right\rangle=\left|\varphi^{o}\right\rangle$ indeed amounts to $\hat{\mathrm{P}}_{\varphi}^{o}$ projection of Eq. 5:

$$
\begin{aligned}
\sum_{i=1}^{m} \hat{\mathrm{P}}_{i}^{b} & =\sum_{s, s^{\prime}}^{o c c d .}\left|s^{o}\right\rangle\left(\sum_{i=1}^{m}\left\langle s^{o} \mid i\right\rangle\left\langle i \mid s^{o}\right\rangle\right)\left\langle s^{\prime o}\left|=\sum_{s, s^{\prime}}^{o c c d .}\right| s^{o}\right\rangle\left(\sum_{i=1}^{m} C_{s, i}^{\dagger} C_{i, s^{\prime}}\right)\left\langle s^{\prime o}\right| \\
& =\sum_{s, s^{\prime}}^{o c c d .}\left|s^{o}\right\rangle \delta_{s, s^{\prime}}\left\langle s^{o}\left|=\sum_{s}^{o c c d .}\right| s^{o}\right\rangle\left\langle s^{o}\right|=\hat{\mathrm{P}}_{\varphi}^{o}
\end{aligned}
$$

where we have recognized the unitary character of the LCAO MO matrix: $\mathbf{C}^{\dagger} \mathbf{C}=\mathbf{I}$.

\section{Through-space and through-bridge bond components}

The square of the off-diagonal CBO matrix element $\gamma_{i, j}$ linking two different AO $\chi_{i}$ and $\chi_{j}$, contributed by atoms $\mathrm{A}$ and $\mathrm{B}$, respectively, determines the ground-state index proposed by Wiberg [8] for the (ground-state) chemical bond-order between these two basis functions:

$$
\begin{aligned}
C \kappa_{i, j} & =\gamma_{i, j} \gamma_{j, i} \\
& =4\left\langle j^{b} \mid i^{b}\right\rangle\left\langle i^{b} \mid j^{b}\right\rangle \\
& =4\left\langle j^{b}\left|\hat{\mathrm{P}}_{i}^{b}\right| j^{b}\right\rangle=2 \gamma_{i, i}\left\langle j^{b}\left|\hat{\overline{\mathrm{P}}}_{i}^{b}\right| j^{b}\right\rangle=4\left|\left\langle i^{b} \mid j^{b}\right\rangle\right|^{2} \equiv 4\left|S_{i, j}\right|^{2} .
\end{aligned}
$$

It constitutes the additive contribution to the overall index of the molecular bondmultiplicity between these atoms,

$$
\mathrm{Cr}_{\mathrm{A}, \mathrm{B}}=\sum_{i \in \mathrm{A}} \sum_{j \in \mathrm{B}} \mathrm{dr}_{i, j} .
$$

This quadratic bond-multiplicity concept has been subsequently extended [9-11], and generalized in terms of the bond-orders from the two-electron difference approach $[1,12-17]$.

It follows from Eq. 3 that this "through-space" dependence between two AO located on different atoms originates from the direct "overlap" $S_{i, j}$ between the bondprojections $\left|i^{b}\right\rangle$ and $\left|j^{b}\right\rangle$ of the two interacting orbitals:

$$
S_{i, j}=\left(\langle i| \hat{\mathrm{P}}_{\varphi}\right)\left(\hat{\mathrm{P}}_{\varphi}|j\rangle\right)=\left\langle i^{b} \mid j^{b}\right\rangle=\gamma_{i, j} / 2
$$

which reflect the overall involvement of these two basis functions in all chemical bonds in the molecular system under consideration.

However, the overall dependence between two $\mathrm{AO}$, say $\chi_{i} \in \mathrm{A}$ and $\chi_{j} \in \mathrm{B}$, in the molecular bond subspace combining all occupied MO has also an indirect ("throughbridge") origins, as represented by the associated amplitude $S_{i, j}$ (bridge). It originates 


$$
\begin{aligned}
& \left\{\left|i^{b}\right\rangle \overrightarrow{s_{i, k}}\left|k^{b}\right\rangle \overrightarrow{s_{k, j}}\left|j^{b}\right\rangle \equiv\left|i^{b}\right\rangle \overrightarrow{s_{i, j}(k)}\left|j^{b}\right\rangle\right\} \\
& \text { 1-bridges } \\
& \left|i^{b}\right\rangle \overrightarrow{s_{i, j}}\left|j^{b}\right\rangle \quad\left\{\left|i^{b}\right\rangle \overrightarrow{s_{i, k}}\left|k^{b}\right\rangle \overrightarrow{s_{k, l}}\left|l^{b}\right\rangle \overrightarrow{s_{l, j}}\left|j^{b}\right\rangle \equiv\left|i^{b}\right\rangle \overrightarrow{s_{i, j}(k, l)}\left|j^{b}\right\rangle\right\} \quad \text { 2-bridges } \\
& \left\{\left|i^{b}\right\rangle \overrightarrow{s_{i, k}}\left|k^{b}\right\rangle \overrightarrow{s_{k, k}}\left|l^{b}\right\rangle \ldots\left|m^{b}\right\rangle \overrightarrow{s_{m, n}}\left|n^{b}\right\rangle \overrightarrow{s_{n, j}}\left|j^{b}\right\rangle \equiv\left|i^{b}\right\rangle \overrightarrow{s_{i, j}(k, l, \ldots, m, n)}\left|j^{b}\right\rangle\right\} \quad t \text {-bridges }
\end{aligned}
$$

Fig. 1 Direct (through-space) and indirect (through-bridges) information propagations between orbitals $\chi_{i}$ and $\chi_{j}$. The latter involve communications through $\alpha=1,3, \ldots, t, \ldots$ AO-intermediaries defining $\alpha$-bridges for the implicit probability scattering in molecules

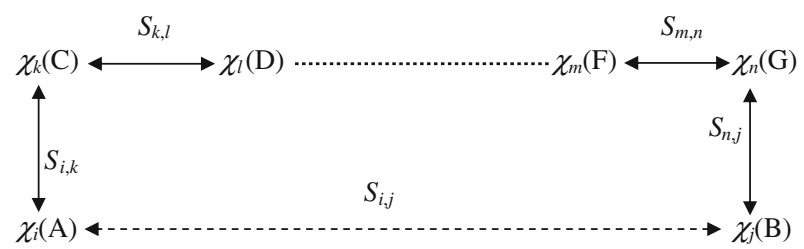

Fig. 2 Direct (through-space) chemical interaction between orbitals $\chi_{i}$ and $\chi_{j}$ (broken line), contributed by atoms $\mathrm{A}$ and $\mathrm{B}$, respectively, and the indirect (through-bridge) interactions (solid lines), through $t \mathrm{AO}$ intermediaries $\left(\chi_{k}, \chi_{l}, \ldots, \chi_{m}, \chi_{n}\right)=\left\{\chi_{r}, r=1,2, \ldots, t\right\}$ contributed by the neighboring bonded atoms $(\mathrm{C}, \mathrm{D}, \ldots, \mathrm{F}, \mathrm{G})$

from the implicit dependences (communications) between two specified AO through the remaining AO in the molecular bonding subspace. As shown in Fig. 1, these intermediate $\mathrm{AO}$ propagations can be classified as originating from the $\mathrm{AO} \alpha$-bridges, $\alpha=1,3, \ldots, t, \ldots$, including $\{\alpha\}$ AO-intermediaries in communications between the specified pair of basis functions:

$$
\begin{aligned}
S_{i, j}(\text { bridge })= & \sum_{k \neq(i, j)} S_{i, j}(k)+\sum_{k, l \neq(i, j)} S_{i, j}(k, l)+\cdots \\
& +\sum_{k, l, \ldots, m, n \neq(i, j)} S_{i, j}(k, l, \ldots, m, n) \\
\equiv & S_{i, j}^{(1)}+S_{i, j}^{(2)}+\cdots+S_{i, j}^{(t)}+\cdots \equiv \sum_{\alpha} S_{i, j}^{(\alpha)} .
\end{aligned}
$$

Clearly, the most important 1-bridge overlaps $\left\{S_{i, j}(k)\right\}$ are through orbitals $\chi_{k} \in \mathrm{C}$ contributed by the atomic neighbor(s) $\mathrm{C}$, chemically bonded to both atoms $\mathrm{A}$ and $\mathrm{B}$, which contribute the specified pair of communicating $\mathrm{AO}$, thus forming atomic bridge A-C-B. Similarly, in a general $t$-bridge the intermediate AO which contribute the most to the overlap $S_{i, j}(k, l, \ldots, m, n)$ between $\chi_{i} \in \mathrm{A}$ and $\chi_{j} \in \mathrm{B}$, through $t$ AO intermediaries (see Fig. 2) $\left(\chi_{k} \in \mathrm{C}, \chi_{l} \in \mathrm{D}, \ldots, \chi_{m} \in \mathrm{F}, \chi_{n} \in \mathrm{G}\right)$, are orbitals contributed by the AIM forming the real bridge of chemical bonds: $\mathrm{A}-\mathrm{C}-\mathrm{D}-\ldots-$ 
$\mathrm{F}-\mathrm{G}-\mathrm{B}$. Such indirect interactions between $\mathrm{A}$ and $\mathrm{B}$ can indeed be long-range in character, provided there exist real chemical bridge connecting the two atoms.

Let us examine the representative $t$-bridge bond-overlap $S_{i, j}(k, l, \ldots, m, n)$ originating from the bond-projections of such $t$ strongly overlapping intermediate AO $\left(\left|k^{b}\right\rangle,\left|l^{b}\right\rangle, \ldots,\left|m^{b}\right\rangle,\left|n^{b}\right\rangle\right)$ (see Fig. 2), contributed by the cluster of the neighboring, bridging atoms $\{\mathrm{C}, \mathrm{D}, \ldots, \mathrm{F}, \mathrm{G}\}$, which connect the specified AIM pair A and B in the molecule. This indirect overlap is proportional to the associated product of the CBO matrix elements:

$$
\begin{aligned}
S_{i, j}(k, l, \ldots, m, n) & =\left\langle i^{b} \mid k^{b}\right\rangle\left\langle k^{b} \mid l^{b}\right\rangle\left\langle l^{b} \mid m^{b}\right\rangle\left\langle m^{b}|\ldots| n^{b}\right\rangle\left\langle n^{b} \mid j^{b}\right\rangle \\
& =2^{-t} \gamma_{i, k} \gamma_{k, l} \gamma_{l, m} \ldots \gamma_{m, n} \gamma_{n, j} .
\end{aligned}
$$

This representative indirect overlap $S_{i, j}(k, l, \ldots, m, n)$, through $t$-bridge, thus constitutes a natural generalization of its direct, through-space analog of Eqs. 10 and 12, by additionally including the product of (non-idempotent) bond-projections onto the indicated intermediate $\mathrm{AO}$,

$$
S_{i, j}(k, l, \ldots, m, n)=\left\langle i^{b}\left|\prod_{r=1}^{t} \hat{\mathrm{P}}_{r}^{b}\right| j^{b}\right\rangle \equiv\left\langle i^{b}\left|\hat{\mathrm{P}}_{t-\text { bridge }}^{b}\right| j^{b}\right\rangle .
$$

In terms of the associated idempotent projectors of Eq. 8 this $t$-bridge overlap reads:

$$
S_{i, j}(k, l, \ldots, m, n)=\left\langle i^{b}\left|\prod_{r=1}^{t}\left(\frac{\gamma_{r, r}}{2}\right) \hat{\overline{\mathrm{P}}}_{r}^{b}\right| j^{b}\right\rangle .
$$

For example, for specific bridges of Fig. 1 these indirect overlaps read:

$$
\begin{aligned}
& S_{i, j}(k)=S_{i, k} S_{k, j}, \quad S_{i, j}(k, l)=S_{i, k} S_{k, l} S_{l, j}, \ldots, \\
& S_{i, j}(k, l, \ldots, m, n)=S_{i, k} S_{k, l} \ldots S_{m, n} S_{n, j}, \ldots
\end{aligned}
$$

Its square defines the associated Wiberg-type bond-order of such an implicit interaction between orbitals $\chi_{i}$ and $\chi_{j}$ originating from this bridge-overlap:

$$
\begin{aligned}
& C \kappa_{i, j}(k, l, \ldots, m, n)=2^{2 t}\left|S_{i, j}(k, l, \ldots, m, n)\right|^{2} \\
& =\gamma_{i, k}\left\{\gamma_{k, l} \ldots\left[\gamma_{m, n}\left(\gamma_{n, j} \gamma_{j, n}\right) \gamma_{n, m}\right] \ldots \gamma_{l, k}\right\} \gamma_{k, i} \\
& =\alpha \kappa_{i, k} \alpha \kappa_{k, l} \alpha \kappa_{l, m} \ldots \alpha \kappa_{m, n} \alpha \kappa_{n, j} \text {. }
\end{aligned}
$$

This indirect bond-multiplicity is thus given by the product of the partial (direct) bondorders of Wiberg, which involve the specified pair of orbitals and all AO intermediaries of the bridge in question. These orbital contributions in turn define the overall interaction between atoms through the specified AO-bridge:

$$
\alpha \kappa_{\mathrm{A}, \mathrm{B}}(k, l, \ldots, m, n)=\sum_{i \in \mathrm{A}} \sum_{j \in \mathrm{B}} \mathrm{Cr}_{i, j}(k, l, \ldots, m, n),
$$


and hence also the overall indirect bond-order due to the given $t$-AIM bridge:

$$
\begin{aligned}
\mathrm{Cr}_{\mathrm{A}, \mathrm{B}}(\mathrm{C}, \mathrm{D}, \ldots, \mathrm{F}, \mathrm{G}) & =\sum_{k \in \mathrm{C}} \sum_{l \in \mathrm{D}} \ldots \sum_{m \in \mathrm{F}} \sum_{n \in \mathrm{G}} \mathrm{cr}_{\mathrm{A}, \mathrm{B}}(k, l, \ldots, m, n) \\
& =\sum_{i \in \mathrm{A}} \sum_{j \in \mathrm{B}} \mathrm{cr}_{i, j}^{(t)}(\mathrm{C}, \mathrm{D}, \ldots, \mathrm{F}, \mathrm{G}) .
\end{aligned}
$$

This bond index explores all implicit dependencies between the bonded atoms A and $\mathrm{B}$, which originate from the basis functions of the $t$ AIM intermediaries defining the atomic bridge in question.

Thus the overall implicit bond-order between atoms A and B:

$$
\begin{aligned}
& \alpha \kappa_{\mathrm{A}, \mathrm{B}}(\text { bridges })=\sum_{k \neq(i, j)} \mathrm{CK}_{i, j}(k)+\sum_{k, l \neq(i, j)} \mathrm{C \kappa _{i,j } ( k , l ) + \cdots} \\
& +\sum_{k, l, \ldots, m, n \neq(i, j)} \propto \kappa_{i, j}(k, l, \ldots, m, n) \\
& \equiv c r_{i, j}^{(1)}+c r_{i, j}^{(2)}+\cdots+c r_{i, j}^{(t)}+\cdots=\sum_{\alpha} d r_{i, j}^{(\alpha)} \text {, }
\end{aligned}
$$

where $C \kappa_{i, j}^{(t)}$ stands for the bond-order generated by all $t$-AIM bridges. Together with the direct component of Eq. 11 this bridge contribution determines the full quadratic bond-multiplicities between atoms in question:

$$
\mathrm{dr}(\mathrm{A}, \mathrm{B})=\alpha r_{\mathrm{A}, \mathrm{B}}+c r_{\mathrm{A}, \mathrm{B}}(\text { bridges }) .
$$

Therefore, the indirect bond component due to AO-bridges can be alternatively viewed in the AIM-resolution defined by the atomic bond-projectors:

$$
\hat{\mathrm{P}}_{\mathrm{X}}^{b}=\sum_{x \in \mathrm{X}}\left|x^{b}\right\rangle\left\langle x^{b}\right|, \mathrm{X}=\mathrm{A}, \mathrm{B}, \mathrm{C}, \ldots
$$

which define the associated AO-bridge projections :

$$
\begin{aligned}
\hat{\mathrm{P}}_{\text {bridges }}^{b} & =\sum_{\mathrm{C} \neq(\mathrm{A}, \mathrm{B})} \hat{\mathrm{P}}_{\mathrm{C}}^{b}+\sum_{\mathrm{C}, \mathrm{D} \neq(\mathrm{A}, \mathrm{B})} \hat{\mathrm{P}}_{\mathrm{C}}^{b} \hat{\mathrm{P}}_{\mathrm{D}}^{b}+\cdots \sum_{\mathrm{C}, \mathrm{D}, \cdots, \mathrm{F}, \mathrm{G} \neq(\mathrm{A}, \mathrm{B})} \hat{\mathrm{P}}_{\mathrm{C}}^{b} \hat{\mathrm{P}}_{\mathrm{D}}^{b} \cdots \hat{\mathrm{P}}_{\mathrm{F}}^{b} \hat{\mathrm{P}}_{\mathrm{G}}^{b}+\cdots \\
& \equiv \sum_{\beta}^{\mathrm{AIM}} \hat{\mathrm{P}}_{\beta-\mathrm{AIM} \text { bridge }}^{b}
\end{aligned}
$$


and the corresponding $\mathrm{A}-\mathrm{B}$ bond-order components:

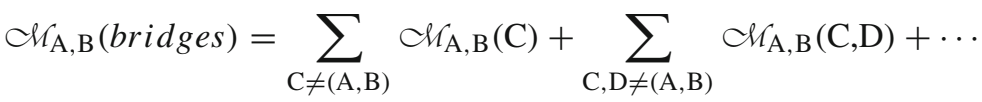

$$
\begin{aligned}
& +\sum_{\mathrm{C}, \mathrm{D}, \ldots, \mathrm{F}, \mathrm{G} \neq(\mathrm{A}, \mathrm{B})} \mathrm{dr} \mathrm{A}, \mathrm{B}_{\mathrm{B}}(\mathrm{C}, \mathrm{D}, \ldots, \mathrm{F}, \mathrm{G}) \\
& \equiv C r_{\mathrm{A}, \mathrm{B}}^{(\mathrm{I})}+c r_{\mathrm{A}, \mathrm{B}}^{(\mathrm{II})}+\cdots+\mathrm{Cr}_{\mathrm{A}, \mathrm{B}}^{(t-\mathrm{AIM})}+\cdots \\
& =\sum_{\beta}^{\mathrm{AIM}} \alpha \mathrm{r}_{\mathrm{A}, \mathrm{B}}^{(\beta-\mathrm{AIM})} \text {. }
\end{aligned}
$$

where:

$$
\begin{aligned}
\mathrm{Cr}_{\mathrm{A}, \mathrm{B}}^{(t-\mathrm{AIM})} & =\left|S_{(\mathrm{A}, \mathrm{B})}(\mathrm{C}, \mathrm{D}, \ldots, \mathrm{F}, \mathrm{G})\right|^{2}=\sum_{i \in \mathrm{A}} \sum_{j \in \mathrm{B}}\left|\left\langle i^{b}\left|\prod_{\mathrm{X}=1}^{t} \hat{\mathrm{P}}_{\mathrm{X}}^{b}\right| j^{b}\right\rangle\right|^{2} \\
& \equiv \sum_{i \in \mathrm{A}} \sum_{j \in \mathrm{B}}\left|\left\langle i^{b}\left|\hat{\mathrm{P}}_{t-\text { AIMbridge }}^{b}\right| j^{b}\right\rangle\right|^{2}
\end{aligned}
$$

This atomic resolution generates contributions better suited for interpretations in chemistry by expressing the overall bridge bond-order $\mathrm{CN}_{\mathrm{A}, \mathrm{B}}$ (bridges) in terms of clusters of AIM, with dominant contributions from $t$-AIM bridges of the chemically bonded bridging atoms connecting $\mathrm{A}$ and $\mathrm{B}$.

Consider now the AO bond-order contributions due to the 1-AIM bridges. One observes that $\sum_{\mathrm{C}} \hat{\mathrm{P}}_{\mathrm{C}}^{b}=\hat{\mathrm{P}}_{\varphi}^{o}$ and hence $\sum_{\mathrm{C} \neq(\mathrm{A}, \mathrm{B})} \hat{\mathrm{P}}_{\mathrm{C}}^{b}=\hat{\mathrm{P}}_{\varphi}^{o}-\hat{\mathrm{P}}_{\mathrm{A}}^{b}-\hat{\mathrm{P}}_{\mathrm{B}}^{b}$.

$$
\begin{aligned}
c r_{\mathrm{A}, \mathrm{B}}^{(\mathrm{I})} & =\sum_{\mathrm{C} \neq(\mathrm{A}, \mathrm{B})} d \hat{\mathrm{A}}_{\mathrm{B}, \mathrm{B}}(\mathrm{C}) \\
& =4 \sum_{i \in \mathrm{A}} \sum_{j \in \mathrm{B}}\left|\left\langle i^{b}\left|\sum_{\mathrm{C} \neq(\mathrm{A}, \mathrm{B})} \hat{\mathrm{P}}_{\mathrm{C}}^{b}\right| j^{b}\right\rangle\right|^{2} \\
& =4 \sum_{i \in \mathrm{A}} \sum_{j \in \mathrm{B}}\left|\left\langle i^{b}\left|\hat{\mathrm{P}}_{\varphi}^{o}-\hat{\mathrm{P}}_{\mathrm{A}}^{b}-\hat{\mathrm{P}}_{\mathrm{B}}^{b}\right| j^{b}\right\rangle\right|^{2} .
\end{aligned}
$$

Recognizing that $\hat{\mathrm{P}}_{\varphi}^{o}\left|\chi^{b}\right\rangle=\left(\hat{\mathrm{P}}_{\varphi}^{o}\right)^{2}|\chi\rangle=\hat{\mathrm{P}}_{\varphi}^{o}|\chi\rangle=\left|\chi^{b}\right\rangle \hat{\mathrm{P}}_{\varphi}^{o}$ and taking into account the AO orthogonality, $\left(\hat{\mathrm{P}}_{\mathrm{A}}^{b}+\hat{\mathrm{P}}_{\mathrm{B}}^{b}\right)\left|j^{b}\right\rangle=\hat{\mathrm{P}}_{\mathrm{B}}^{b}\left|j^{b}\right\rangle=\left|j^{b}\right\rangle \gamma_{j, j} / 2$ and $\left(\hat{\mathrm{P}}_{\mathrm{A}}^{b}+\hat{\mathrm{P}}_{\mathrm{B}}^{b}\right)\left|i^{b}\right\rangle=$ $\hat{\mathrm{P}}_{\mathrm{A}}^{b}\left|i^{b}\right\rangle=\left|i^{b}\right\rangle \gamma_{i, i} / 2$, gives:

$$
\alpha r_{\mathrm{A}, \mathrm{B}}^{(\mathrm{I})}=4 \sum_{i \in \mathrm{A}} \sum_{j \in \mathrm{B}}\left|\left\langle i^{b}\left|\hat{\mathrm{P}}_{\varphi}^{o}-\hat{\mathrm{P}}_{\mathrm{A}}^{b}-\hat{\mathrm{P}}_{\mathrm{B}}^{b}\right| j^{b}\right\rangle\right|^{2}
$$




$$
\begin{aligned}
& =4 \sum_{i \in \mathrm{A}} \sum_{j \in \mathrm{B}}\left\langle j^{b}\left|\hat{\mathrm{P}}_{\varphi}^{o}-\hat{\mathrm{P}}_{\mathrm{A}}^{b}-\hat{\mathrm{P}}_{\mathrm{B}}^{b}\right| i^{b}\right\rangle\left\langle i^{b}\left|\hat{\mathrm{P}}_{\varphi}^{o}-\hat{\mathrm{P}}_{\mathrm{A}}^{b}-\hat{\mathrm{P}}_{\mathrm{B}}^{b}\right| j^{b}\right\rangle \\
& =4 \sum_{i \in \mathrm{A}} \sum_{j \in \mathrm{B}}\left|S_{i, j}\right|^{2}\left[1-\frac{1}{2}\left(\gamma_{i, i}+\gamma_{j, j}\right)+\frac{1}{4} \gamma_{i, i} \gamma_{j, j}\right] \\
& =\alpha \hat{\mathrm{A}}_{\mathrm{B}, \mathrm{B}}-\frac{1}{4} \sum_{i \in \mathrm{A}} \sum_{j \in \mathrm{B}} \mathrm{d \gamma _{i,j }}\left[2\left(\gamma_{i, i}+\gamma_{j, j}\right)-\gamma_{i, i} \gamma_{j, j}\right] .
\end{aligned}
$$

This equation determines the exact relation between the indirect $\mathrm{A}-\mathrm{B}$ bond-order, realized through single-AIM bridges of all remaining atoms, $\mathrm{Cr}_{\mathrm{A}, \mathrm{B}}^{(\mathrm{I})}$, and the direct through-space Wiberg component $\mathcal{C K}_{\mathrm{A}, \mathrm{B}}$ of the overall bond-multiplicity of Eq. 20.

Let us examine two limiting occupations of AO on atoms A and B. For the full occupations in the molecule of all AO contributed by these two atoms, $\left\{\gamma_{i, i}=\gamma_{j, j}=2\right\}$, i.e., $\left\{2\left(\gamma_{i, i}+\gamma_{j, j}\right)-\gamma_{i, i} \gamma_{j, j}=4\right\}$, when they remain effectively non-bonded, exhibiting only the lone-pairs in their inner and valence shells, Eq. 26 gives: $c r_{\mathrm{A}, \mathrm{B}}^{(\mathrm{I})}=$ $C \kappa_{\mathrm{A}, \mathrm{B}}-\alpha \kappa_{\mathrm{A}, \mathrm{B}}=0$. Therefore, as intuitively expected, the non-bonded AIM do not generate the implicit bond-component through the single-AIM bridges. Indeed, since the bridge-projectors probe the common part of the diatomic bonding subspace $\left\{\left|\chi_{\mathrm{A}}^{b}\right\rangle,\left|\chi_{\mathrm{B}}^{b}\right\rangle\right\}$ and the remaining basis functions $\left\{\left|\chi_{\mathrm{C} \neq(\mathrm{A}, \mathrm{B})}^{b}\right|\right\}$, for non-bonded $\mathrm{A}$ and $\mathrm{B}$, which do not exhibit the common part of the AO subspace with any single remaining atoms and hence also with AO bases of any cluster of such bridging atoms, one predicts the vanishing bond-order contribution generated through any bridges: atomic, diatomic, triatomic, etc. It should be stressed that the common bonding subspaces for a larger number of AIM should steadily decrease with increasing order of the AIMcluster in bridges, thus implying their expected diminished contribution to the overall bridge-component of the chemical bond under consideration: $\mathrm{Cr}_{\mathrm{A}, \mathrm{B}}^{(\mathrm{I})}>\mathrm{Cr}_{\mathrm{A}, \mathrm{B}}^{(\mathrm{II})}>\ldots$ The bridging atoms must be also mutually bonded to generate the appreciable through bridge overlap of the interacting AO [see Eq. 14], so that significant hypothetical bridges are in fact limited to real chemical bridges of atoms in the molecular structural formula.

The next configuration of interest is the one in which all orbitals from A and B are half-occupied in the molecule: $\left\{\gamma_{i, i}=\gamma_{j, j}=1\right\}$, i.e., $\left\{2\left(\gamma_{i, i}+\gamma_{j, j}\right)-\gamma_{i, i} \gamma_{j, j}=3\right\}$. This implies that $\left|\chi_{\mathrm{A}}\right\rangle$ and $\left|\chi_{\mathrm{B}}\right\rangle$ are heavily engaged in forming the chemical bonds

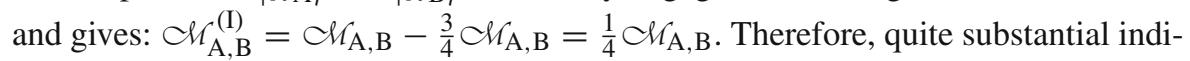
rect bond-order, amounting to a quarter of the through-space component, is realized already through all single-atomic bridges in the molecule. It should be also realized that in this case one also expects non-vanishing contributions from higher orders of AIM bridges.

\section{Conditional probabilities for information propagation}

The key concept of Communication Theory of the Chemical Bond (CTCB) is the molecular information system [1,2]. It can be constructed at alternative levels of 
resolving the electron probabilities into the underlying electron-localization "events", which determine the channel inputs $\boldsymbol{a}=\left\{a_{i}\right\}$ and outputs $\boldsymbol{b}=\left\{b_{j}\right\}$. In OCT the AO basis functions of SCF MO calculations determine a natural resolution level for discussing the information contributions to the multiplicity (order) of the system chemical bonds: $\boldsymbol{a}=\left\{\chi_{i}\right\}$ and $\boldsymbol{b}=\left\{\chi_{j}\right\}$. The AO networks describe the probability/information propagation in the molecule, which can be described by the standard quantities developed in IT for real communication devices [30-33]. Due to electron delocalization throughout the network of chemical bonds the transmission of "signals" about the electron-assignments to AO becomes randomly disturbed, thus exhibiting typical communication "noise". Indeed, an electron initially attributed to the given AO in the channel "input" $\boldsymbol{a}$ can be later found with a non-zero probability at several locations in the molecular "output" $\boldsymbol{b}$. This feature of the electron delocalization is embodied in the conditional probabilities of the "outputs-given-inputs", $\mathbf{P}(\boldsymbol{b} \mid \boldsymbol{a})=\left\{P\left(\chi_{j} \mid \chi_{i}\right) \equiv P(j \mid i)\right\}$, which define the molecular information network.

In OCT one constructs the orbital-pair probabilities [2,7,26-29] using the superposition-principle of quantum mechanics [34] supplemented by the "physical" projection onto the subspace of the system occupied MO, which determine the molecular network of chemical bonds. The AIM off-diagonal orbital communications are then related to Wiberg's [8] bond-order contributions or the generalized "quadratic" bond multiplicities [9-17] formulated in MO theory. The IT descriptors have been shown to account for the chemical intuition quite well, at the same time providing the resolution of the diatomic bond-multiplicities into their complementary IT-covalent and IT-ionic components $[2,7,35]$.

The orbital information system involves the AO events in the channel input $\boldsymbol{a}=\left\{\chi_{i}\right\}$ and output $\boldsymbol{b}=\left\{\chi_{j}\right\}$. In this description the $\mathrm{AO} \rightarrow \mathrm{AO}$ communication network is determined by the conditional probabilities

$$
\mathbf{P}(\boldsymbol{b} \mid \boldsymbol{a})=\left\{P(j \mid i)=P(i \wedge j) / p_{i}\right\}, \quad \sum_{j} P(j \mid i)=1,
$$

where the joint probabilities of simultaneously observing two AO in the system chemical bonds $\mathbf{P}(\boldsymbol{a} \wedge \boldsymbol{b})=\{P(i \wedge j)\}$ satisfy the usual normalization relations:

$$
\sum_{i} P(i \wedge j)=p_{j}, \quad \sum_{j} P(i \wedge j)=p_{i}, \quad \sum_{i} \sum_{j} P(i \wedge j)=1 .
$$

As argued elsewhere [2,27] these conditional probabilities for the direct (throughspace) communications between $\mathrm{AO}$ involve squares of corresponding off-diagonal elements of the CBO matrix; for the closed-shell systems one obtains:

$$
\mathbf{P}(\boldsymbol{b} \mid \boldsymbol{a})=\left\{P(j \mid i)=\left(2 \gamma_{i, i}\right)^{-1} \gamma_{i, j} \gamma_{j, i}=\left(2 \gamma_{i, i}\right)^{-1}\left(\gamma_{i, j}\right)^{2}\right\}
$$


The associated joint-probability matrix,

$$
\begin{aligned}
& \mathbf{P}(\boldsymbol{a} \wedge \boldsymbol{b})= \\
& \quad\left\{P(i \wedge j)=p_{i} P(j \mid i)=(2 N)^{-1} \gamma_{i, j} \gamma_{j, i}=(2 N)^{-1}\left\langle i\left|\hat{\mathrm{P}}_{\varphi}\right| j\right\rangle\left\langle j\left|\hat{\mathrm{P}}_{\varphi}\right| i\right\rangle\right\},
\end{aligned}
$$

indeed satisfies the normalization conditions of Eq. 28, e.g.,

$$
\sum_{i} P(i \wedge j)=(2 N)^{-1} \sum_{i} \gamma_{j, i} \gamma_{i, j}=(2 N)^{-1} 2 \gamma_{j, j}=p_{j}
$$

It should be finally observed that the conditional probability of Eq. 29 has the following simple interpretation in terms of the AO projectors of Eq. 8:

$$
P(j \mid i)=\frac{2}{\gamma_{i, i}}\left\langle j^{b}\left|\hat{\mathrm{P}}_{i}^{b}\right| j^{b}\right\rangle=\left\langle j^{b}\left|\hat{\mathrm{P}}_{i}^{b}\right| j^{b}\right\rangle .
$$

In order to estimate the IT bond contributions due to the through-bridge interactions between the specified pair of basis functions, in the molecular input and output, respectively, one requires the associated conditional probabilities realized through the indicated AO intermediaries. A straightforward projection-generalization of the preceding expression gives:

$$
\begin{aligned}
& P[(j \mid i) \mid k, l, \ldots, m, n]=\left\langle j^{b}\left|\left(\hat{\overline{\mathrm{P}}}_{n}^{b} \hat{\overline{\mathrm{P}}}_{m}^{b} \ldots \hat{\overline{\mathrm{P}}}_{l}^{b} \hat{\overline{\mathrm{P}}}_{k}^{b}\right) \hat{\overline{\mathrm{P}}}_{i}^{b}\left(\hat{\overline{\mathrm{P}}}_{k}^{b} \hat{\overline{\mathrm{P}}}_{l}^{b} \ldots \hat{\overline{\mathrm{P}}}_{m}^{b} \hat{\overline{\mathrm{P}}}_{n}^{b}\right)\right| j^{b}\right\rangle \\
& \equiv\left\langle j^{b}\left|\hat{\overline{\mathrm{P}}}_{\text {bridge }}^{b \dagger} \hat{\overline{\mathrm{P}}}_{i}^{b} \hat{\overline{\mathrm{P}}}_{\text {bridge }}^{b}\right| j^{b}\right\rangle \\
& =\frac{1}{2 \gamma_{i, i} \gamma_{k, k}^{2} \gamma_{l, l}^{2} \ldots \gamma_{m, m}^{2} \gamma_{n, n}^{2}} \gamma_{i, k}\left\{\gamma_{k, l} \ldots\left[\gamma_{m, n}\left(\gamma_{n, j} \gamma_{j, n}\right) \gamma_{n, m}\right] \ldots \gamma_{l, k}\right\} \gamma_{k, i} \\
& =\frac{1}{2 \gamma_{i, i}}\left(\frac{\gamma_{i, k} \gamma_{k, l} \ldots \gamma_{m, n} \gamma_{n, j}}{\gamma_{k, k} \gamma_{l, l} \ldots \gamma_{m, m} \gamma_{n, n}}\right)^{2} \\
& \equiv P[(j \mid i) \mid t-\mathrm{AO}] \text {. }
\end{aligned}
$$

For example, for the single-AO bridge $\chi_{k}$ one obtains

$$
P[(j \mid i) \mid k]=\frac{1}{2 \gamma_{i, i}}\left(\frac{\gamma_{i, k} \gamma_{k, j}}{\gamma_{k, k}}\right)^{2},
$$

while two-AO intermediaries $\left(\chi_{k}, \chi_{l}\right)$ give

$$
P[(j \mid i) \mid k, l]=\frac{1}{2 \gamma_{i, i}}\left(\frac{\gamma_{i, k} \gamma_{k, l} \gamma_{l, j}}{\gamma_{k, k} \gamma_{l, l}}\right)^{2} .
$$

These conditional probabilities determine the effective through-bridge communications between the specified AO-input $\left(\chi_{i}\right)$ and AO-otput $\left(\chi_{j}\right)$, thus defining the associated information systems for each order of the AO or AIM bridges. In OCT 
the entropy/information indices of the covalent/ionic components of chemical bonds represent the complementary descriptors of the average amounts of the information scattered (communication noise) and the the information conserved (information-flow) in the molecular communication channel of interest. The purely molecular communication channel, devoid of any reference (history) of the chemical bond formation process, uses the molecular AO probabilities $\boldsymbol{p}=\left\{p_{i}=\gamma_{i, i} / N\right\}$ in the channel input, while the promolecular signal $\boldsymbol{p}^{0}=\left\{p_{i}^{0}=\gamma_{i, i}^{0} / N\right\}$, reflectiong the ground-state electron configurations of the collection of the constituent free atoms, is used to extract the ionic IT-component of the resultant IT-bond order [1,2].

Thus, the average (through-space) noise index of the molecular IT bond-covalency is measured by the conditional-entropy of the system outputs given inputs, in accordance with probabilities of Eqs. 29 and 32,

$$
S(\boldsymbol{b} \mid \boldsymbol{a})=\sum_{i} p_{i}\left[-\sum_{j} P(j \mid i) \log P(j \mid i)\right] \equiv S(\boldsymbol{p} \mid \boldsymbol{p})=S .
$$

The AO channel with the promolecular input "signal" $\boldsymbol{p}^{0}$ refers to the initial-state in the bond-formation process. It corresponds to the ground-state (fractional) occupations of the AO contributed by the system constituent (free) atoms, before their mixing into MO. These input probabilities give rise to the average information-flow descriptor of the system IT bond-ionicity, given by the mutual-information in the channel inputs and outputs:

$$
\begin{aligned}
& \boldsymbol{I}\left(\boldsymbol{a}^{0}: \boldsymbol{b}\right)= \\
& \quad \sum_{i} p_{i}\left\{\sum_{j} P(j \mid i) \log \left[P(i \mid j) / p_{i}^{0}\right]\right\} \equiv \boldsymbol{I}\left(\boldsymbol{p}^{0}: \boldsymbol{p}\right)=I=H\left(\boldsymbol{p}^{0}\right)-S,
\end{aligned}
$$

where $H\left(\boldsymbol{p}^{0}\right)=-\sum_{i} p_{i}^{0} \log p_{i}^{0}$. This amount of information reflects the fraction of the initial (promolecular) information content $S\left(p^{0}\right)$, which has not been dissipated as noise in the molecular communication system. The sum of these two bond components,

$$
\mathcal{\gamma}\left(\boldsymbol{a}^{0} ; \boldsymbol{b}\right) \equiv \mathcal{\gamma}\left(\boldsymbol{p}^{0} ; \boldsymbol{p}\right)=H\left(\boldsymbol{p}^{0}\right)
$$

measures the overall direct IT-multiplicity of all bonds in the molecular system under consideration. It is conserved at the promolecular-entropy level $H\left(\boldsymbol{p}^{0}\right)$ which marks the initial information content of AO probabilities. The flexible-input approach to localized chemical interactions is also available, which reproduces the Wiberg bondorders in diatomics [2,7].

The implicit IT-covalent and IT-ionic components of chemical bonds, realized through the intermediate propagations of $\mathrm{AO}$ probabilities through bridges, can 
be defined in a similar way for any order of the AO/AIM bridge. For example, for the given $t$-AO bridge $(k, l, \ldots, m, n)$ of Fig. 2 the conditional probabilities $P[(j \mid i) \mid k, l, \ldots, m, n]$ determine the associated through-bridge communications between the input $(i)$ and output $(j) \mathrm{AO}$, and their average noise-component,

$$
\begin{aligned}
S[(\boldsymbol{b} \mid \boldsymbol{a}) \mid k, l, \ldots, m, n] & =-\sum_{i} \sum_{j} P[(i \wedge j) \mid k, l, \ldots, m, n] \log P[(j \mid i) \mid k, l, \ldots, m, n] \\
& \equiv S(k, l, \ldots, m, n) .
\end{aligned}
$$

where $P[(i \wedge j) \mid k, l, \ldots, m, n]=p_{i} P[(j \mid i) \mid k, l, \ldots, m, n]$, reflects the system extra, indirect IT-covalency generated by this particular AO-bridge.

\section{Illustrative application to $\pi$-electron systems in benzene and butadiene}

Next, let us examine the indirect $\pi$-bonds between carbon atoms in benzene and butadiene by using the familiar Hückel approximation. The $\mathrm{CBO}$ matrix in benzene is summarized by the following elements:

$$
\gamma_{i, i}=1, \quad \gamma_{i, i+1}=2 / 3, \quad \gamma_{i, i+2}=0, \quad \gamma_{i, i+3}=-1 / 3
$$

thus generating the associated direct (through-space) bond-components of Wiberg:

$$
d \kappa_{i, i+1}=0.44, \quad d \kappa_{i, i+2}=0, \quad d \kappa_{i, i+3}=0.11 .
$$

The vanishing direct bond-orders between the meta-carbons is supplemented by the complementary indirect interactions through the real $\pi$-bond system:

$$
\begin{aligned}
& \mathrm{CK_{i,i+2 }}(i+1)=\mathrm{Ch}_{i, i+1} \mathrm{CK_{i+1,i+2 }}=0.20, \\
& \mathrm{d \kappa _{i,i+2 }}(i+5, i+4, i+3)=\mathrm{d \kappa _{i,i+5 }} \mathrm{Cr}_{i+5, i+4} \mathrm{Cr}_{i+4, i+3} \mathrm{Cr}_{i+3, i+2}=0.04 \text {, }
\end{aligned}
$$

which together amount to the overall 0.24 indirect $\pi$-bond multiplicity between meta carbons realized through the remaining (neighboring) $\mathrm{C}-\mathrm{C}$ bonds in benzene. There also are two small Wiberg-type contributions due to bridges involving non-neighbors in the ring:

$$
\begin{aligned}
& \alpha \kappa_{i, i+2}(i+3)=\alpha \kappa_{i, i+3} \mathrm{Cr}_{i+3, i+2}=\mathrm{CK_{i,i+2 }}(i+5) \\
& =\mathrm{C} \kappa_{i, i+5} \mathrm{C} \kappa_{i+5, i+2}=0.05 \text {, } \\
& \mathrm{Cr}_{i, i+2}(i+1, i+4, i+3)=\mathrm{Cr}_{i, i+1} \mathrm{Ch}_{i+1, i+4} \mathrm{Cr}_{i+4, i+3} \mathrm{Cr}_{i+3, i+2} \\
& =\mathrm{Ch}_{i, i+2}(i+1, i+4, i+5) \\
& =\mathrm{C} \kappa_{i, i+1} \mathrm{C} \kappa_{i+1, i+4} \mathrm{C} \kappa_{i+4, i+5} \mathrm{CK_{i+5,i+2 }}=0.01 \text {. }
\end{aligned}
$$


Therefore, the meta-carbons in benzene, which in this approximation exhibit the vanishing direct (through-space) $\pi$-bond, are linked by the indirect bond multiplicity $\mathrm{C}_{i, i+2}$ (bridges) $\cong 0.3$. In this rough estimate we have neglected some very small contributions due to bridges involving a single or several cross-ring links, e.g.,

$$
\alpha \kappa_{i, i+2}(i+3, i+4, i+5)=\alpha \kappa_{i, i+3} \mathrm{Ch}_{i+3, i+4} \mathrm{Ch}_{i+4, i+5} \mathrm{d \kappa _{i+5,i+2 }}=0.002 .
$$

The neighboring, ortho-carbons, which exhibit the highest direct $\pi$-bond order, generate relatively small overall bridge contribution. All single- and triple-AO bridges generate the vanishing indirect components, while the only non-vanishing contributions result from the following double- and quadruple-AO bridges:

$$
\begin{aligned}
& \alpha \kappa_{i, i+1}(i+5, i+4)=\alpha \kappa_{i, i+5} d \kappa_{i+5, i+4} d K_{i+4, i+1} \\
& =\mathrm{C} \hat{K}_{i, i+1}(i+2, i+3) \\
& =\mathrm{C} \hat{K}_{i+1, i+2} \mathrm{CK_{i+2,i+3 }} \mathrm{Ch}_{i+3, i}=0.02 \text {, } \\
& \mathrm{dr} \gamma_{i, i+1}(i+2, i+3, i+4, i+5)=\mathrm{Cr}_{i+1, i+2} \mathrm{Cr}_{i+2, i+3} \mathrm{Ch}_{i+3, i+4} \mathrm{Cr}_{i+4, i+5} \mathrm{Cr}_{i+5, i} \\
& =0.02 \text {, }
\end{aligned}
$$

generating altogether $d \widehat{N}_{i, i+1}$ (bridges) $=0.06$. This implicit contribution and the direct part of Eq. 41 give rise to the half total bond-order between neighboring carbons in the benzene ring, as intuitively expected. In this estimate we have again neglected very-small contributions generated by bridges involving several para-links in the ring, e.g.,

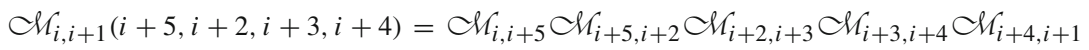

$$
\begin{aligned}
& =0.001 \text {, }
\end{aligned}
$$

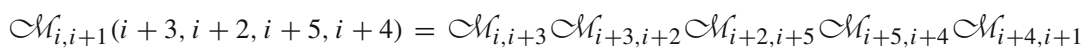

$$
\begin{aligned}
& =0.0004 \text {. }
\end{aligned}
$$

Finally, let us consider the chemical bridge components of $\pi$-interactions between the para-carbons in the ring, which exhibit a relatively small direct bond-order reported in Eq. 41. Again, all single- and triple-AO bridges generate the vanishing indirect components, while the most important double-AO bridges realized through neighboring bonds give:

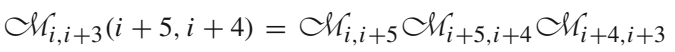

$$
\begin{aligned}
& =d \kappa_{i, i+3}(i+1, i+2)=d \kappa_{i, i+1} d \kappa_{i+1, i+2} d \kappa_{i+2, i+3}=0.09 \text {, }
\end{aligned}
$$


i.e., $C K_{i, i+3}$ (bridges $) \cong 0.18$. There are also two quadruple-AO bridges involving two cross-ring links, which contribute a very small indirect bond-order between para-carbons in benzene:

$$
\begin{aligned}
\mathrm{Ch}_{i, i+3}(i+1, i+4, i+5, i+2) & =\mathrm{Ch}_{i, i+1} \mathrm{Ch}_{i+1, i+4} \mathrm{Ch}_{i+4, i+5} \mathrm{Ch}_{i+5, i+2} \mathrm{Ch}_{i+2, i+3} \\
& =0.001 .
\end{aligned}
$$

Therefore, two para-carbons in the benzene ring exhibit the resultant $\pi$-bond order of Eq. 20 to the amount of about 0.3 , similar to that characterizing two meta-carbons.

The artificial distinction of the cross-ring interactions in Wiberg's (direct) multiplicities, with the vanishing bond-order for meta-carbons, is thus removed when the through-bridges components are taken into account:

$$
\operatorname{ch}(\text { para }) \cong \operatorname{Cr}(\text { meta })=0.3<\operatorname{Cr}(\text { ortho })=0.5 \text {. }
$$

We again emphasize the differences in their compositions: the para interactions exhibit comparable through-space and through-bridge components, the meta multiplicities are realized exclusively through bridges, while the strongest ortho bond-orders have practically direct, through-space origins.

Of interest also is a comparison of the bond-order contributions realized through the ring bridges of increasing length:

$$
\begin{aligned}
& C K_{i, i+2}(i+1)=\left(C K_{i, i+1}\right)^{2}=0.20 ; \quad C K_{i, i+3}(i+1, i+2)=\left(C K_{i, i+1}\right)^{3}=0.09 ; \\
& \mathrm{Ch}_{i, i+4}(i+1, i+2, i+3)=\left(\mathrm{Cr}_{i, i+1}\right)^{4}=0.04 \text {; } \\
& C \kappa_{i, i+5}(i+1, i+2 i+3, i+4)=\left(\mathrm{CK_{i,i+1 }}\right)^{5}=0.02 \text {. }
\end{aligned}
$$

Let us examine next the $\pi$-interactions in butadiene. For the consecutive numbering of carbon atoms the off-diagonal part of the CBO matrix in Hückel approximation is fully characterized by the following elements:

$$
\gamma_{1,2}=\gamma_{3,4}=2 / \sqrt{5}, \quad \gamma_{1,3}=\gamma_{2,4}=0, \quad \gamma_{1,4}=-1 / \sqrt{5}, \quad \gamma_{2,3}=1 / \sqrt{5}
$$

which determine the associated through-space bond-orders:

$$
d r_{1,2}=d r_{3,4}=0.80, \quad d r_{1,3}=d r_{2,4}=0, \quad d r_{1,4}=d r_{2,3}=0.20 .
$$

This rather artificial distinction of the (1-3) and (2-4) interactions as non-bonding can be expected to be again remedied by the inclusion of the indirect bond components. For the strongest, terminal bond (1-2) the only non-vanishing contribution is due to two-AO bridge

$$
\alpha \kappa_{1,2}(4,3)=\alpha \kappa_{1,4} d \kappa_{4,3} \alpha \kappa_{3,2}=0.03=\alpha \kappa_{1,2} \text { (bridges), }
$$


Fig. 3 Indirect (through-bridges)

communication system for the effective $\pi$-interactions between two meta-carbons in the benzene ring

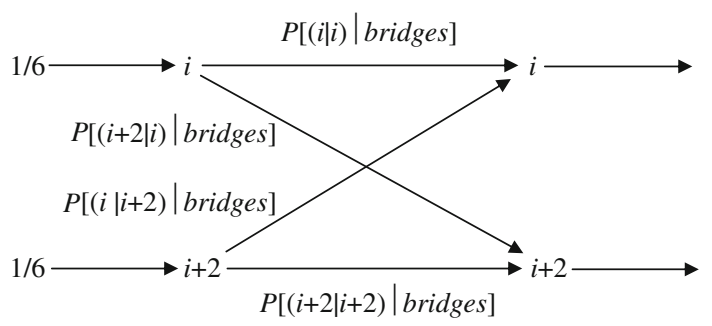

and hence the resultant bond order of terminal bonds:

$$
\alpha(1-2)=\operatorname{cr}(3-4)=0.83 .
$$

The bridge contributions to the second-neighbor interactions (1-3) read:

$$
d r_{1,3}(4)=d r_{1,4} d r_{4,3}=d r_{1,3}(2)=d r_{1,2} d r_{2,3}=0.16,
$$

and hence

$$
\mathrm{Cr}_{1,3}(\text { bridges })=0.32=\mathrm{ch}(1-3) .
$$

The bridge contribution to $\pi$-interactions between terminal carbon atoms,

$$
d \kappa_{1,4}(2,3)=d \kappa_{1,2} d r_{2,3} d r_{3,4}=0.13=\alpha r_{1,4}(\text { bridges }),
$$

and between carbons of the middle bond,

$$
d \kappa_{2,3}(1,4)=\alpha \kappa_{2,1} d \kappa_{1,4} d \kappa_{4,3}=0.13=\alpha \kappa_{1,3} \text { (bridges), }
$$

finally give:

$$
\operatorname{dr}(1,4)=\operatorname{cr}(2,3)=0.33 \text {. }
$$

Therefore, this novel perspective on $\pi$-bond multiplicities in butadiene, more rational than that following from the direct bond-orders of Wiberg, predicts:

$$
\begin{aligned}
\operatorname{cr}(1-2) & =\operatorname{cr}(3-4)=0.83>\{\operatorname{ch}(1-4)=\operatorname{ch}(2-3)=0.33 \cong \operatorname{cr}(1-3) \\
& =\operatorname{cr}(2-4)=0.32\} .
\end{aligned}
$$

The strongest, terminal bonds (1-2) and (3-4) are almost exclusively of the throughspace origin, the $\pi$-bonds (1-3) and (2-4) connecting the second-neighbors exhibit the pure through-bridge character, while the remaining bonds (1-4) and (2-3) include comparable direct and indirect components. 

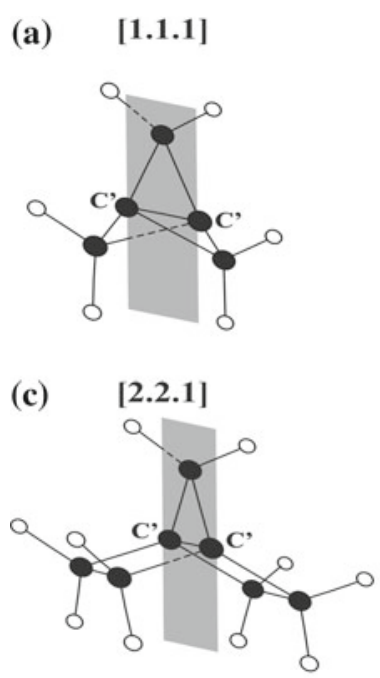

(b) [2.1.1]

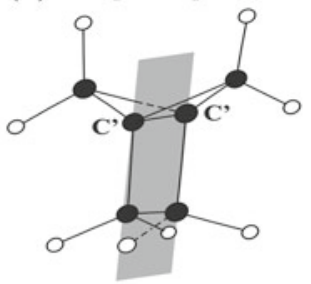

(d) $[2.2 .2]$

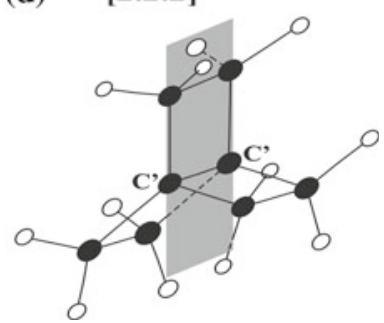

Fig. 4 Propellane structures and the planes of sections containing the bridge and bridgehead $\left(\mathrm{C}^{\prime}\right)$ carbon atoms identified by black circles

\section{Orbital communications}

In this section we shall briefly examine the through-bridge communications in benzene focusing on the indirect probabilities determining the communication system for the directly non-interacting meta-carbons in the benzene ring. We recall, that the symmetry-unrelated, direct conditional probabilities in benzene,

$$
P(i \mid i)=1 / 2, \quad P(i+1 \mid i)=2 / 9, P(i+2 \mid i)=0, \quad P(i+3 \mid i)=1 / 18,
$$

define the through-space AO-communications for this molecule.

For the most important meta-bridges of Eqs. 42-44 one finds the following offdiagonal probabilities linking these two carbon atoms through bridges:

$$
\begin{aligned}
& P[(i+2 \mid i) \mid i+1]=0.0988, \quad P[(i+2 \mid i) \mid i+3]=0.0247, \\
& P[(i+2 \mid i) \mid i+5, i+4, i+3]=0.0195,
\end{aligned}
$$

and hence

$$
P[(i+2 \mid i) \mid \text { bridges }]=P[(i \mid i+2) \mid \text { bridges }] \cong 0.1430 .
$$


[1.1.1]

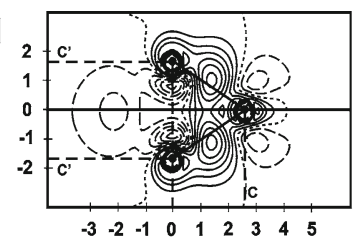

[2.1.1]

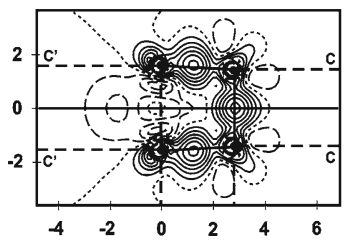

[2.2.1]

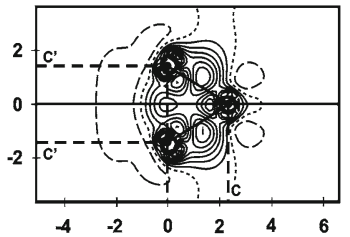

[2.2.2]

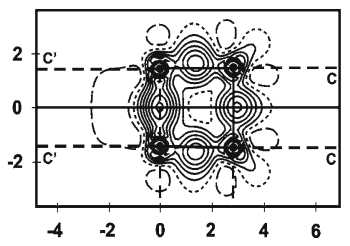

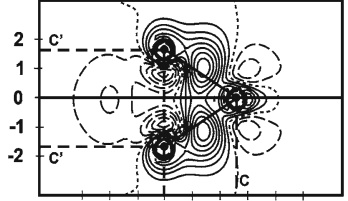

$\begin{array}{ccccccccc}-3 & -2 & -1 & 0 & 1 & 2 & 3 & 4 & 5\end{array}$
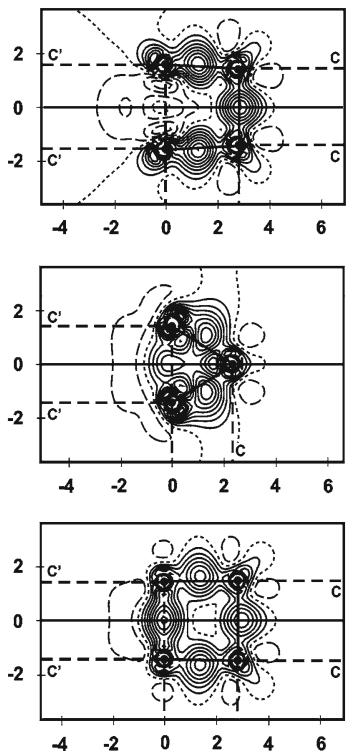
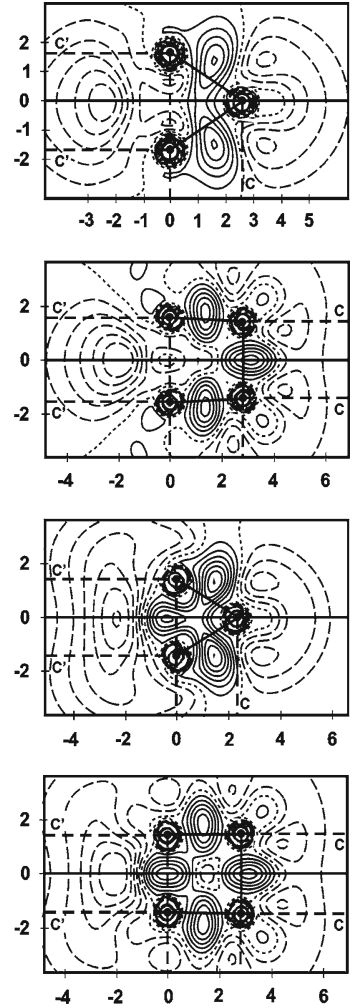

Fig. 5 A comparison between the equidistant-contour maps of the density-difference function $\Delta \rho(\boldsymbol{r})=$ $\rho(\boldsymbol{r})-\rho^{0}(\boldsymbol{r})$ between the molecular $(\rho)$ and promolecular $\left(\rho^{0}\right)$ electron densities (first column), the information-distance density $\Delta s(\boldsymbol{r})=\rho(\boldsymbol{r}) \ln \left[\rho(\boldsymbol{r}) / \rho^{0}(\boldsymbol{r})\right]$ (second column), and the Shannon entropy displacement density $h_{\rho}(\boldsymbol{r})=\rho^{0}(\boldsymbol{r}) \ln \rho^{0}(\boldsymbol{r})-\rho(\boldsymbol{r}) \ln \rho(\boldsymbol{r})$ (third column), for the four propellanes of Fig. 4. The solid, pointed and broken lines denote the positive, zero and negative values, respectively of the equally spaced contours

One similarly estimates the corresponding bridge contributions for the diagonal probabilities realized through these three bridges,

$$
\begin{aligned}
& P[(i \mid i) \mid i+1]=P[(i+2 \mid i+2) \mid i+1]=P[(i+2 \mid i+2) \mid i+3]=0.0988, \\
& P[(i \mid i) \mid i+3]=0.0062 \\
& P[(i \mid i) 1 / 2 i+5, i+4, i+3]=P[(i+2 \mid i+2) \mid i+5, i+4, i+3]=0.0049,
\end{aligned}
$$

which give:

$$
P[(i \mid i) \mid \text { bridges }] \cong 0.110 \text { and } P[(i+2 \mid i+2) \mid \text { bridges }] \cong 0.202
$$



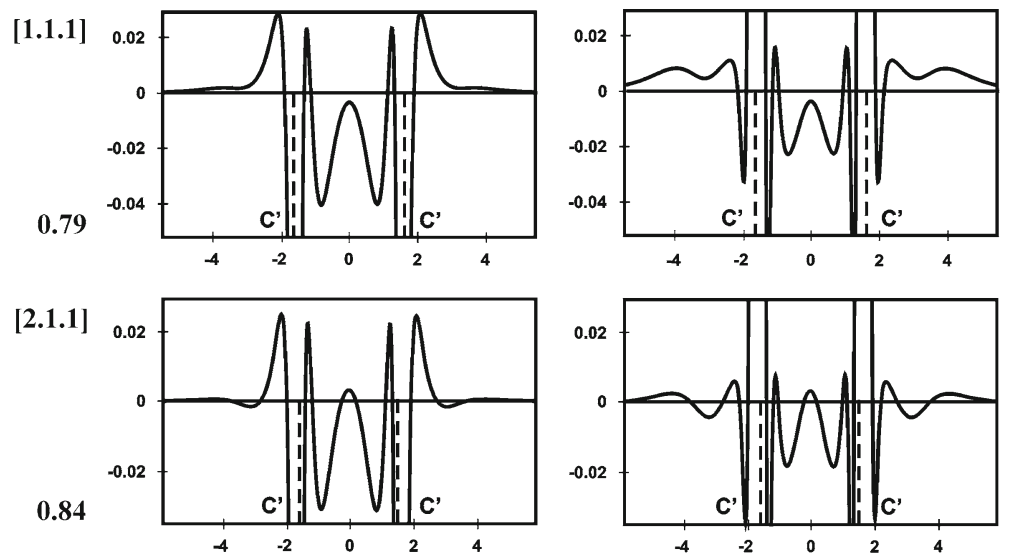

[2.2.1]
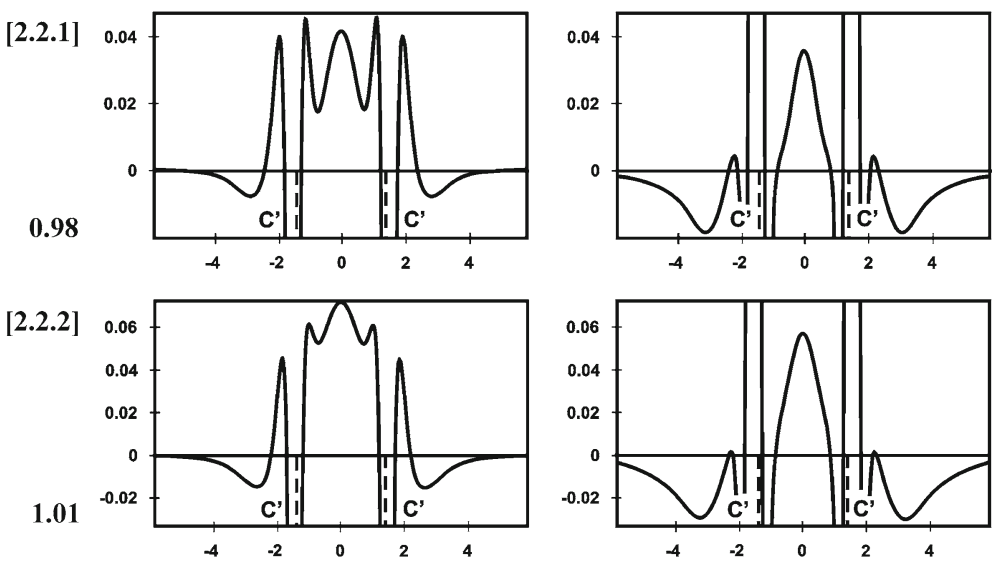

Fig. 6 The bridgehead bond profiles of the density difference function (left panel) and molecular entropy displacement (right panel) for the four propellanes of Fig. 3. For comparison the numerical values of the bond multiplicities [6] from the two-electron difference approach [1,12-17] are also reported

Resultant probabilities of Eqs. 67 and 69 then define the indirect-communication channel for the indirect $\pi$-interactions between the meta carbons in benzene ring shown in Fig. 3. Its average conditional entropy (communication noise), which measures the implicit IT-covalency of the $\pi$-bond-order between the meta-carbons in the benzene ring, realized through these three bridges: $S[(i+2 \mid i) \mid$ bridges $] \cong 0.27$ (bits). This IT estimate is close to the associated Wiberg-type indirect bond multiplicity $C \kappa_{i, i+2}($ bridges $) \cong 0.3$ reported in the preceding section.

\section{Central bonds in propellanes}

As already mentioned in Sect. 1, the existence of the through-bridges chemical bonds has been first conjectured in an attempt to explain the bond-order indices for the smallest propellanes of Fig. 4 obtained from the two-electron difference approach $[1,2,6]$. First, we briefly summarize these results. 
The density-difference diagrams, contour maps of alternative local-information probes and ELF plots for these molecules all testify to the absence of the constructive interference between orbitals of the central carbons in the smallest $[1,1,1]$ and $[2,1,1]$ propellanes [1-3,21,23,25]. It eventually appears in the two largest [2,2,1] and [2,2,2] systems, in which at least two bridges contain two carbon atoms. This is explicitly shown in Figs. 5 and 6 reporting the density difference and information-density contour maps and profiles, respectively [1-3].

However, thermodynamical data for these systems and previously reported quadratic, two-electron bond-order indices [6] reported in Fig. 6, both confirm the existence of some central bond, even in the smallest of these systems. This prompted alternative propositions of the through-bridges [1-3] and VB-inspired charge-shift [36] mechanisms, with the latter attributing this bonding effect to instantaneous charge fluctuations, to explain the apparent existence of some chemical bonding between the central carbons in the smallest propellanes, despite the absence of the charge accumulation between central carbons in these molecules. The bond-orders of Fig. 6 roughly estimate a full (single) central bond in the [2,2,1] and [2,2,2] propellanes, while that in smaller $[2,1,1]$ and $[1,1,1]$ systems, lacking the through-space component, stays at about 0.8 level. Thus, by attributing the latter estimate to the through-bridges component, one predicts about 0.2 bond-order measure attributed to the complementary through-space part of the central $\mathrm{C}-\mathrm{C}$ bond [3].

\section{Conclusion}

We have explored in this work the through-bridge mechanism of bonding interactions in molecular systems, which has been first conjectured to explain the numerical bond orders for propellane systems. In the Wiberg-type bond-order description this indirect bonding interaction, realized through the orbital/AIM intermediaries called "bridges", has been shown to rationalize the bonding patterns in propellanes. It also gives an additional insight into the $\pi$-bonding in benzene and butadiene, by removing some artifacts of the traditional Wiberg bond-order description, e.g., of trends in the cross-ring interactions. It has been shown to bring the overall interactions of the two meta-carbons, lacking the direct (through-space) component, to approximately the same level of about 0.3 bond as that predicted for the two carbons in the mutual para-positions. The same extra IT-covalency follows from the OCT using the conditional-probability corrections due to bridges. The dominant ortho-interactions in benzene and terminal bonds in butadiene have been shown to be almost exclusively of the through-space character, while the second-neighbor interactions in butadiene and meta interactions in benzene were found to be of the purely through-bridges origin. This novel mechanism adds to the complexity/diversity of chemical interactions in molecular systems, and it offers an alternative perspective on some controversial chemical bonds in molecules, e.g., on the central bond problem in propellanes. It also removes some artifacts of the over-simplified approach based solely upon the through-space mechanism.

Open Access This article is distributed under the terms of the Creative Commons Attribution Noncommercial License which permits any noncommercial use, distribution, and reproduction in any medium, provided the original author(s) and source are credited. 


\section{References}

1. R.F. Nalewajski, Information Theory of Molecular Systems (Elsevier, Amsterdam, 2006)

2. R.F. Nalewajski, Information Origins of the Chemical Bond (Nova, New York, 2010)

3. R.F. Nalewajski, E. Broniatowska, J. Phys. Chem. A 107, 6270 (2003)

4. R.F. Nalewajski, E. Świtka, A. Michalak, Int. J. Quantum. Chem. 87, 198 (2002)

5. R.F. Nalewajski, E. Świtka, Phys. Chem. Chem. Phys. 4, 4952 (2002)

6. R.F. Nalewajski, J. Mrozek, G. Mazur, Can. J. Chem. 100, 1121 (1996)

7. R.F. Nalewajski, D. Szczepanik, J. Mrozek, Adv. Quant. Chem. (in press)

8. K.A. Wiberg, Tetrahedron 24, 1083 (1968)

9. M.S. Gopinathan, K. Jug, Theor. Chim. Acta (Berl.) 63, 497, 511 (1983)

10. K. Jug, M.S. Gopinathan, in Theoretical Models of Chemical Bonding, vol. II, ed. by Z.B. Maksić (Springer, Heidelberg, 1990), p. 77

11. I. Mayer, Chem. Phys. Lett. 97, 270 (1983)

12. R.F. Nalewajski, A.M. Köster, K. Jug, Theoret. Chim. Acta (Berl.) 85, 463 (1993)

13. R.F. Nalewajski, J. Mrozek, Int. J. Quantum Chem. 51, 187 (1994)

14. R.F. Nalewajski, S.J. Formosinho, A.J.C. Varandas, J. Mrozek, Int. J. Quantum Chem. 52, 1153 (1994)

15. R.F. Nalewajski, J. Mrozek, A. Michalak, Int. J. Quantum Chem. 61, 589 (1997)

16. J. Mrozek, R.F. Nalewajski, A. Michalak, Polish J. Chem. 72, 1779 (1998)

17. R.F. Nalewajski, Chem. Phys. Lett. 386, 265 (2004)

18. A.D. Becke, K.E. Edgecombe, J. Chem. Phys. 92, 5397 (1990)

19. B. Silvi, A. Savin, Nature 371, 683 (1994)

20. A. Savin, R. Nesper, S. Wengert, T.F. Fässler, Angew. Chem. Int. Ed. Engl. 36, 1808 (1997)

21. R.F. Nalewajski, A.M. Köster, S. Escalante, J. Phys. Chem. A 109, 10038 (2005)

22. R.F. Nalewajski, Int. J. Quantum Chem. 108, 2230 (2008)

23. R.F. Nalewajski, P. de Silva, J. Mrozek, in Kinetic Energy Functional, ed. by A. Wang, T. Wesołowski (World Scientific, Singapore, 2009), (in press)

24. R.F. Nalewajski, J. Math. Chem. 47, 667 (2010)

25. R.F. Nalewajski, P. de Silva, J. Mrozek, J. Mol. Struct. THEOCHEM. 954, 57 (2010)

26. R.F. Nalewajski, Adv. Quant. Chem. 56, 217 (2009)

27. R.F. Nalewajski, Int. J. Quantum Chem. 109, 425, 2495 (2009)

28. R.F. Nalewajski, J. Math. Chem. 47, 692, 808 (2010)

29. R.F. Nalewajski, J. Math. Chem. doi:10.1007/s10910-010-9766-3

30. C.E. Shannon, Bell Syst. Tech. J. 27, 379, 623 (1948)

31. C.E. Shannon, W. Weaver, The Mathematical Theory of Communication (University of Illinois, Urbana, 1949)

32. N. Abramson, Information Theory and Coding (McGraw-Hill, New York, 1963)

33. P.E. Pfeifer, Concepts of Probability Theory, 2nd edn. (Dover, New York, 1978)

34. P.A.M. Dirac, The Principles of Quantum Mechanics, 4th edn. (Clarendon, Oxford, 1958)

35. R.F. Nalewajski, J. Math. Chem. 47, 709 (2010)

36. S. Shaik, D. Danovich, W. Wu, P.C. Hiberty, Nat. Chem. 1, 443 (2009) 